\title{
Alignment of tracer gradient vectors in 2D turbulence
}

\author{
Patrice Klein ${ }^{\mathrm{a}, \mathrm{b}, *}$, Bach Lien Hua ${ }^{\mathrm{b}, \mathrm{a}}$, Guillaume Lapeyre ${ }^{\mathrm{b}, \mathrm{a}}$ \\ ${ }^{a}$ Laboratoire de Physique des Océans, IFREMER, BP 70, 29280 Plouzané, France \\ ${ }^{\mathrm{b}}$ Laboratoire de Météorologie Dynamique, 75005 Paris, France
}

Received 20 January 2000; received in revised form 10 April 2000; accepted 26 May 2000

Communicated by U. Frisch

\begin{abstract}
This numerical study examines the stirring properties of a 2D flow field with a specific focus on the alignment dynamics of tracer gradient vectors. In accordance with the study of Hua and Klein [Physica D 113 (1998) 98], our approach involves the full second order Lagrangian dynamics and in particular the second order in time equation for the tracer gradient norm. If the physical space is partitioned into strain-dominated regions and "effective" rotation-dominated regions (following a criterion defined by Lapeyre et al. [Phys. Fluids 11 (1999) 3729]), the new result of this study concerns the "effective" rotation-dominated regions: it is found, from numerical simulations of 2D turbulence, that the tracer gradient vector statistically aligns with one of the eigenvector of a tensor that comes out from the second order equation and is related to the pressure Hessian. The consequence is that, in those regions, the observed exponential growth or decay of the tracer gradient vector can be predicted contrary to previous results which implied zero growth and only a rotation of this vector. This result strongly emphasizes the important role of the time evolution of the strain rate amplitude which, with the rotation of the strain tensor, significantly contributes to the alignment dynamics. Both effects are related to the anisotropic part of the pressure Hessian, which emphasizes the non-locality of the mechanisms involved. These results are reminiscent of those recently obtained by Nomura and Post [J. Fluid Mech. 377 (1998) 65] for 3D turbulence. (c) 2000 Elsevier Science B.V. All rights reserved.
\end{abstract}

Keywords: Tracer gradient vectors; 2D turbulence; Pressure Hessian

\section{Introduction}

It is well known that the properties of large-scale geophysical flows can be understood through the study of $2 \mathrm{D}$ turbulent flow fields. Within this context one important issue concerns their stirring and mixing properties [21]. The question is how to identify the regions of physical space where the turbulent cascade is the most active through the production of tracer gradients. To address this question, several studies have focused on the deformation of a tracer field through the dynam-

\footnotetext{
* Corresponding author.

E-mail address: patrice.klein@ifremer.fr (P. Klein).
}

ics of its gradients (e.g. [3,9-11,15,19,24,25]). This approach has also been intensively used for 3D turbulent flow field to understand the accumulation of vorticity in thin sets (e.g. $[8,18]$ and references therein).

For 2D turbulence, Okubo [19] and Weiss [24] (see also [25]) were the first to derive a kinematic criterion, based on the first order equation for the gradient vector, to separate "straining regions" where the motions strongly shear the vorticity or a passively advected tracer from "eddy regions" where motions advect the vorticity or tracer smoothly. However, counterexamples, such as the point vortex flow, suffice to show that this criterion is not generally indicative of the growth rate of the gradients [20]. An alternative to examine the 
tendency towards a turbulent cascade is to consider the first order equation for the tracer gradient norm that involves the magnitude of the strain rate (known in terms of the velocity field) and the geometrical alignment of the tracer gradient vector with the eigenvectors of the strain rate tensor $[15,22]$. Thus the effectiveness of the turbulent cascade crucially depends on the alignment properties which need to be estimated.

One interesting property of the alignment dynamics, which stimulates our study, is that the orientation of the tracer gradient vector appears to be determined only by its recent history [2,4]. This means that over a few strain rate scales, an equilibrium should be attained. Some studies [17,22] have revealed a tendency for the vorticity gradient vector to align with the compressional strain eigenvector. Lapeyre et al. [13] have recently revisited the question of the existence of a preferential direction for the tracer gradient. Their results show that the alignment dynamics is a response to a competition between the strain effects and the "effective" rotation (i.e. the sum of vorticity and rotation of the axes of the strain rate tensor) effects. This has led to define a criterion to separate strain-dominated regime from "effective" rotation-dominated regime. Their results represent a substantial improvement to the Okubo-Weiss criterion since an exact analytical solution is found for the class of axisymmetric vortices (which the point vortex belongs to) that lies between these two regimes. Furthermore, for the strain-dominated regime, they proposed an exact steady solution for the alignment which is distinct from the compressional eigenvector of the strain tensor. This solution was shown to predict more accurately the tracer gradient orientation than those involving only the compressional eigenvector of the strain tensor. However, for the "effective" rotation-dominated regime, their solution predicts no growth of the tracer gradient, which was found to be less statistically robust.

The original point of Lapeyre et al. [13] is to take into account explicitly the rotation of the axes of the strain rate tensor. This quantity cannot be deduced directly from the local velocity field whereas the vorticity and strain rate are expressed in terms of the local velocity field. Actually this quantity is a non-local quantity which requires to consider the Lagrangian acceleration gradient tensor (equal to the pressure Hessian for a $2 \mathrm{D}$ flow), i.e. to consider the second order Lagrangian dynamics. This emphasizes the non-local character of the stirring properties and the important role of the pressure Hessian that has already been highlighted in [3,9]. This role of the pressure Hessian on the stirring properties has also been well emphasized by recent analytical and numerical results in 3D turbulence (e.g. [8,18,23]). However, the solutions derived in [13] use only a part of the information contained in the pressure Hessian since they do not involve the time evolution of the magnitude of the strain rate. Our conjecture, based on our previous studies in $2 \mathrm{D}$ turbulence [9] and on more recent results in 3D turbulence [16], is that the full consideration of the pressure Hessian should improve the solutions already proposed, in particular for the "effective" rotation-dominated regions. Indeed Fig. 18 of [16] shows that in the rotation-dominated regions of a $3 \mathrm{D}$ turbulent flow field the gradient vector aligns preferentially with one of the eigenvectors of the pressure Hessian.

Consequently this paper reexamines the alignment properties of tracer gradients in a 2D flow field with the consideration of the full second order Lagrangian dynamics by using the first two order time derivatives equations for the tracer gradient. A rationalization of this approach, based on a Taylor series expansion, has been given by Ohkitani and Kishiba [18]. Following the recent results of Nomura and Post [16], a specific purpose of our study is to investigate whether the eigenvectors of the tensor involved in the second order equation represent a preferential direction for the tracer gradient vector in the "effective" rotation-dominated regime. Our approach is developed and discussed in the next two sections. Its potentialities are assessed in Section 4 from numerical experiments of 2D decaying turbulence. For the sake of simplicity, our approach is developed within the context of the 2D Euler equations since the addition of viscosity makes the analytical problem difficult to interpret. However, the numerical experiments result from the integration of the Navier-Stokes equations with the inclusion of a Newtonian viscosity. The conclusions are summarized in Section 5. 


\section{Second order Lagrangian approach of alignment dynamics}

Let us consider a tracer field $q$ conserved on a Lagrangian trajectory:

$\frac{\mathrm{d} q}{\mathrm{~d} t}=0$,

where $(\mathrm{d} / \mathrm{d} t)()=\partial() / \partial t+u(\partial() / \partial x)+v(\partial() / \partial y)$, with $u$ and $v$, respectively, the zonal and meridional components of the $2 \mathrm{D}$ velocity field.

The approach used in [9] to study the deformation of the tracer field was to consider the equations of the first and second orders in time for the tracer gradient vector. The first equation involves the velocity gradient tensor and the second one the pressure Hessian (see Appendix A). In this study, we use a slightly different approach: we consider the first two order time derivative equations for the norm of the tracer gradient instead of those for the gradient vector. The main argument for this choice is that these equations are independent of the coordinates system and allow to focus on the geometrical alignment of the tracer gradient vector. Defining $|\nabla q|^{2} \equiv \nabla q^{*} \nabla q$, with $*$ denoting the transpose, we obtain

$\frac{\mathrm{d}}{\mathrm{d} t}|\nabla q|^{2}=-2 \nabla q^{*} \boldsymbol{S} \nabla q$,

$\frac{\mathrm{d}^{2}}{\mathrm{~d} t^{2}}|\nabla q|^{2}=\nabla q^{*} N \nabla q$

with

$\boldsymbol{S}=\frac{1}{2}\left[\begin{array}{cc}\sigma_{\mathrm{n}} & \sigma_{\mathrm{s}} \\ \sigma_{\mathrm{s}} & -\sigma_{\mathrm{n}}\end{array}\right]$,

$\boldsymbol{N}=\left[\begin{array}{cc}\sigma_{\mathrm{n}}^{2}+\sigma_{\mathrm{s}}^{2}-\omega \sigma_{\mathrm{s}}-\frac{\mathrm{d} \sigma_{\mathrm{n}}}{\mathrm{d} t} & \omega \sigma_{\mathrm{n}}-\frac{\mathrm{d} \sigma_{\mathrm{s}}}{\mathrm{d} t} \\ \omega \sigma_{\mathrm{n}}-\frac{\mathrm{d} \sigma_{\mathrm{s}}}{\mathrm{d} t} & \sigma_{\mathrm{n}}^{2}+\sigma_{\mathrm{s}}^{2}+\omega \sigma_{\mathrm{s}}+\frac{\mathrm{d} \sigma_{\mathrm{n}}}{\mathrm{d} t}\end{array}\right]$,

where $\omega, \sigma_{\mathrm{n}}$ and $\sigma_{\mathrm{s}}$ are, respectively, the vorticity and the normal and shear strain rates defined as $\omega \equiv$ $\partial v / \partial x-\partial u / \partial y, \sigma_{\mathrm{n}} \equiv \partial u / \partial x-\partial v / \partial y$ and $\sigma_{\mathrm{s}} \equiv$ $\partial v / \partial x+\partial u / \partial y$. Note that in $2 \mathrm{D}$ turbulence, the vorticity $\omega$ is a tracer that verifies (2.1).

Eq. (2.2) involves the strain tensor $S$ and Eq. (2.3) the tensor $\boldsymbol{N}$. The tensor $\boldsymbol{N}$ involves $\mathrm{d} \boldsymbol{S} / \mathrm{d} t$ and is

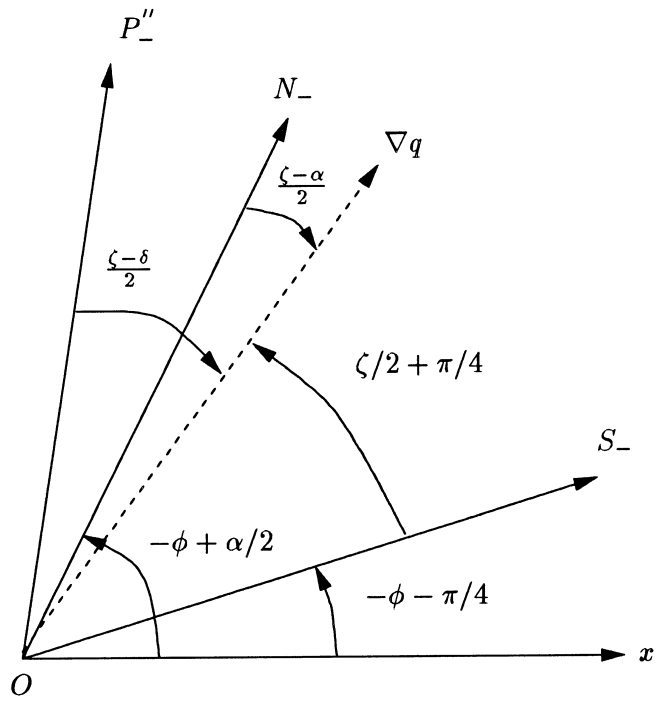

Fig. 1. Angles between $\nabla q$ and the different eigenvectors. The different angles are defined in the text.

directly related to the pressure Hessian (see Appendix A). Let us introduce $\boldsymbol{S}_{-}$and $\boldsymbol{N}_{-},{ }^{1}$ the eigenvectors of, respectively, $S$ and $\boldsymbol{N}$ corresponding to their lowest eigenvalues. From (2.2), the growth rate of the tracer gradient depends on the eigenvalues of $\boldsymbol{S}$ and on the alignment of $\boldsymbol{\nabla} q$ with $\boldsymbol{S}_{-}$. On the other hand, from (2.3) (see [8] for a more complete demonstration), the growth rate tendency and the dynamics of the alignment of $\nabla q$ with one of the eigenvectors of $S$ depend on the alignment of $\nabla q$ with $\boldsymbol{N}_{-}$. Thus $\boldsymbol{N}$ explicitly affects the alignment dynamics. Consequently when $\nabla q$ does not align with $\boldsymbol{S}_{-}$, as in the "effective" rotation-dominated regime (see [13]), it seems natural to examine whether it preferentially aligns with $\boldsymbol{N}_{-}$.

A way to examine more explicitly the alignment dynamics is to rewrite (2.2) and (2.3) using the notations $\nabla q=\rho(\cos \theta, \sin \theta)$ with $\rho \geq 0$ (see Fig. 1) and $\left(\sigma_{\mathrm{s}}, \sigma_{\mathrm{n}}\right)=\sigma(\cos 2 \phi, \sin 2 \phi)$ with $\sigma \geq 0$. These equations become

$\frac{1}{\rho^{2}} \frac{\mathrm{d}}{\mathrm{d} t} \rho^{2}=-\sigma \sin \zeta$

$\frac{1}{\rho^{2}} \frac{\mathrm{d}^{2}}{\mathrm{~d} t^{2}} \rho^{2}=\sigma^{2}(1-\chi \cos (\zeta-\alpha))$,

\footnotetext{
${ }^{1} \boldsymbol{N}$ (as well as $\boldsymbol{S}$ ) is symmetric and therefore its eigenvectors (and those of $\boldsymbol{S}$ ) are orthogonal. So $\boldsymbol{S}_{-}$and $\boldsymbol{N}_{-}$suffice to characterize the eigenvectors of $S$ and $N$.
} 
with

$\zeta=2(\theta+\phi)$

and where $\chi$ and $\alpha$ are defined by

$\chi=\sqrt{r^{2}+s^{2}}, \quad(\sin \alpha, \cos \alpha)=\left(\frac{s}{\chi}, \frac{r}{\chi}\right)$,

with

$r \equiv \frac{\omega+2(\mathrm{~d} \phi / \mathrm{d} t)}{\sigma}, \quad s \equiv \frac{\mathrm{d} \sigma / \mathrm{d} t}{\sigma^{2}}$.

The angles $\theta, \phi, \zeta$ and $\alpha$ are shown in Fig. 1 .

The RHS of (2.6), i.e. the exponential growth rate of the tracer gradient norm, involves only $\sigma$ and $\zeta$. The angle $\zeta$ is directly related to the orientation of $\nabla q$ with the compressional eigenvector $\boldsymbol{S}_{-}$, since from the preceding definitions the angle of $S_{-}$with the $x$-axis is $-(\phi+\pi / 4)$ (see Fig. 1). Then a perfect alignment of $\nabla q$ with the compressional eigenvector $(\zeta=-\pi / 2)$ corresponds to the largest growth rate. On the other hand, $\zeta=0$ means that $\nabla q$ is aligned with the bisector of the strain rate eigenvectors, which leads to a zero exponential growth rate. The RHS of (2.7) involves two new non-dimensional terms: $r$ and $s$. The term $r$ is a "rotational" term related to the "effective" rotation (i.e. the rotation effects resulting from the competition between the vorticity and the rotation of the main axes of the strain rate tensor). The term $s$ represents the effects of the time evolution of the strain rate amplitude. It should be emphasized that both tensors, $\boldsymbol{N}$ and $\mathrm{d} \boldsymbol{S} / \mathrm{d} t$ (and hence the anisotropic part of the pressure Hessian), can be expressed in terms of $r$ and $s$ (see Appendix B).

From Eqs. (2.2) and (2.3), $\boldsymbol{S}_{-}$and $\boldsymbol{N}_{-}$represent two natural directions which the tracer gradient vector could preferentially align with. The angle between $S_{-}$and $\boldsymbol{N}_{-}$is just equal to $\frac{1}{2}(\alpha+\pi / 2)$ (Fig. 1). This means that, when both $r$ and $s$ differ from zero, $N_{-}$ is coaligned neither with the strain axes nor with the bisector of the strain axes. The alignment of $\nabla q$ with $\boldsymbol{S}_{-}$has been addressed by several studies (see [22] and references therein). However, a more recent study [13] has shown that the tracer gradient vectors prefer to align with specific directions that differ from $S_{-}$. These directions, that involve only the value of the parameter $r$ (and not $s$ ), were derived by using only the first order equation for the tracer gradient vector. The present study, based on the first and second order equations for the tracer gradient norm, yields a new direction, $\boldsymbol{N}_{-}$, which naturally stems from the second order equation and that depends on both $r$ and $s$. Consequently the question now is: do the tracer gradient vectors prefer to align with $\boldsymbol{N}_{-}$rather than with the other directions? This question is addressed in the next sections within the framework of Eqs. (2.2) and (2.3).

\section{Discussion of the approach used}

\subsection{Relation with previous work}

The combination of (2.6) and (2.7) allows to obtain an equation for the time evolution of the angle $\zeta$ :

$\frac{\mathrm{d} \zeta}{\mathrm{d} t}=\sigma(r-\cos \zeta)$

The equation is actually identical to that derived by Lapeyre et al. [13]. Similar equations have also been derived by Dresselhaus and Tabor [6] and Dritschel et al. [7] (see also [12]). Eq. (3.1) only involves $r$ and has led Lapeyre et al. [13] to define two regimes: the strain-dominated regime (when $|r| \leq 1$ ) for which there are two steady solutions:

$\zeta_{-}=-\arccos (r)$ stable,

$\zeta_{+}=\arccos (r)$ unstable,

and the "effective" rotation-dominated regime (when $|r|>1$ ) for which no steady solution exists. Steady solutions express that the alignment angle, $\zeta$, results from the balance between the strain effects $(\sigma)$ that tend to align the tracer gradient vector in the direction of the compressional eigenvector $\boldsymbol{S}_{-}$and the rotation effects $(\omega+2(\mathrm{~d} \phi / \mathrm{d} t))$ that tend to move away the tracer gradient vector from $S_{-}$.

For the "effective" rotation-dominated regime $(|r|>1)$, Lapeyre et al. [13] only predicted a continuous rotation of the gradient vector with a minimum rate of rotation for a preferential orientation

$\zeta_{\text {prob }}=\frac{\pi}{2}(1-\operatorname{sign}(r))$ 
which corresponds to the bisector of the strain axes and therefore to a zero exponential growth rate of the tracer gradient norm.

The solutions of Lapeyre et al. [13] depend only on $r$. However, Eqs. (2.6) and (2.7) reveal that both $r$ and $s$ should affect the alignment dynamics and the growth rate. Consequently we would like to examine, in particular for the "effective" rotation-dominated regime, whether the consideration of the full second order Lagrangian dynamics, allows to better identify the preferential direction for the alignment of the tracer gradient. More precisely we intend to examine whether the eigenvector $\boldsymbol{N}_{-}$represents a preferential direction for the tracer gradient vectors or not. Some insights for a positive answer are given by the solutions for the alignment dynamics for three different limiting cases.

\subsection{Three limiting cases}

These limiting cases concern the saddle point, the axisymmetric vortex and the strongly rotating case.

1. The saddle point $(r=0)$. For a saddle point where $r=s=0(\chi=0)$, the stable solutions of (2.6) and (2.7) lead to

$$
\sin \zeta=-\frac{C_{1} \sinh (\sigma t)+C_{2} \cosh (\sigma t)}{C_{1} \cosh (\sigma t)+C_{2} \sinh (\sigma t)}
$$

where $C_{1}$ and $C_{2}$ are constants. This yields the following equilibrated solution $\zeta=-\pi / 2$. Consequently the exponential growth rate of the tracer gradient is maximum and $\nabla q$ is exactly aligned with the compressional eigenvector $S_{-}$. Furthermore, $\nabla q$ is also exactly aligned with $\boldsymbol{N}_{-}$(since $\boldsymbol{N}$ is a diagonal matrix and therefore any vector is an eigenvector, which emphasizes the degenerated character of this example).

2. The axisymmetric vortex $(|r|=1)$. A steady axisymmetric vortex is characterized by a stream function that is only radially varying. (Note that the point vortex satisfies this characteristic.) This leads to $r= \pm 1$ and $s=0(\chi=1)$. Then the exact solutions of Eqs. (2.6) and (2.7) lead to

$$
\sin \zeta=-2 r \frac{A+r \sigma t}{1+(A+r \sigma t)^{2}}
$$

where $A$ is a constant. At equilibrium, $\zeta=0$ or $\pi$, which means that the exponential growth rate of the tracer gradient is 0 and that the vector $\nabla q$ exactly aligns with the bisector of the strain axes. The equilibrated solution corresponds to only an algebraic growth. However, since $\alpha=\arctan (s / r)=0, \nabla q$ is exactly aligned with $\boldsymbol{N}_{-}$.

3. The strongly rotating case $(|r| \gg 1)$. From (3.1) there is no steady solution for $\zeta$ in that case and the tracer gradient is continuously rotating. However, one can argue (as in [13]) that the tracer gradient vector could spend statistically more time in the direction for which $|\mathrm{d} \zeta / \mathrm{d} t|$ is minimum (i.e. for $\mathrm{d}^{2} \zeta / \mathrm{d} t^{2} \approx 0$ ). This direction can be found from the equation for $\mathrm{d}^{2} \zeta / \mathrm{d} t^{2}$ only assuming that $2\left|\mathrm{~d}^{2} \phi / \mathrm{d} t^{2}\right| \ll|\mathrm{d} \sigma / \mathrm{d} t|$, which leads to

$\frac{\mathrm{d}^{2} \zeta}{\mathrm{d} t^{2}}=\sigma^{2} \chi \sin (\zeta-\alpha)$.

Thus the preferential direction that corresponds to $\mathrm{d}^{2} \zeta / \mathrm{d} t^{2} \approx 0$ is $\zeta \approx \alpha$. This direction that strongly depends on $s$ corresponds to $\boldsymbol{N}_{-}$. It differs from the strain axes and from the bisector of the strain axes when $s$ is non-zero.

These specific examples that are only limiting cases are quite different with respect to the alignment of $\nabla q$ with the eigenvectors of $\boldsymbol{S}$. The first one has a degenerated character. However in the two other examples, that represent the lower and upper bounds of the "effective" rotation-dominated regime, $\nabla q$ aligns with $N_{-}$. Furthermore, the last one involves both parameters $r$ and $s$ and takes into account strong unsteadiness. This suggests that, in a $2 \mathrm{D}$ time-evolving turbulent flow field, $\nabla q$ could be preferentially aligned with the eigenvector $N_{-}$, in particular for the "effective" rotation-dominated regime. Consequently, in order to assess the importance of the parameters $r$ and $s$, the next section examines, from the results of numerical experiments, the alignment of the tracer gradient vector with various directions.

\section{Experimental results}

The experimental results concern the flow field obtained in a numerical simulation of decaying turbu- 
lence at a resolution of $1024 \times 1024$ (see [9] for more details). There is a Newtonian viscosity such that the Reynolds number is $3.5 \times 10^{4}$. In the following sections, the characteristics of this flow field are analyzed after about 40 turnover time scales of the predominant eddies. At this stage of the decay, the vorticity field (see [9, Fig. 1]) exhibits the usual emergence of coherent structures together with a strong filamentation resulting from the mutual straining and shearing influences of closely located vortices. The results discussed below have been corroborated by the analysis (not reported here) of the numerical simulation at later times (after 50 and 60 turnover time scales) and also of other numerical simulations of 2D turbulence.

\subsection{Preferential alignment of the tracer gradient vector}

The preferential direction which the vector $\nabla q$ tends to align with has been estimated through the statistics of the geometrical alignment of $\nabla q$ with different vectors. The vectors considered are, first, the compressional eigenvector $\boldsymbol{S}_{-}$and the eigenvector $\boldsymbol{N}_{-}$. The alignment of $\nabla q$ with $\boldsymbol{S}_{-}$(respectively, $\boldsymbol{N}_{-}$) is assessed by checking whether $\zeta \approx-\pi / 2$ (respectively, $\zeta \approx \alpha$ ). Furthermore, we have also estimated the alignment with the solutions of Lapeyre et al. [13] namely, the steady solution (3.2) when $|r|<1$ (by checking whether $\zeta \approx \zeta_{-}$) and the most probable

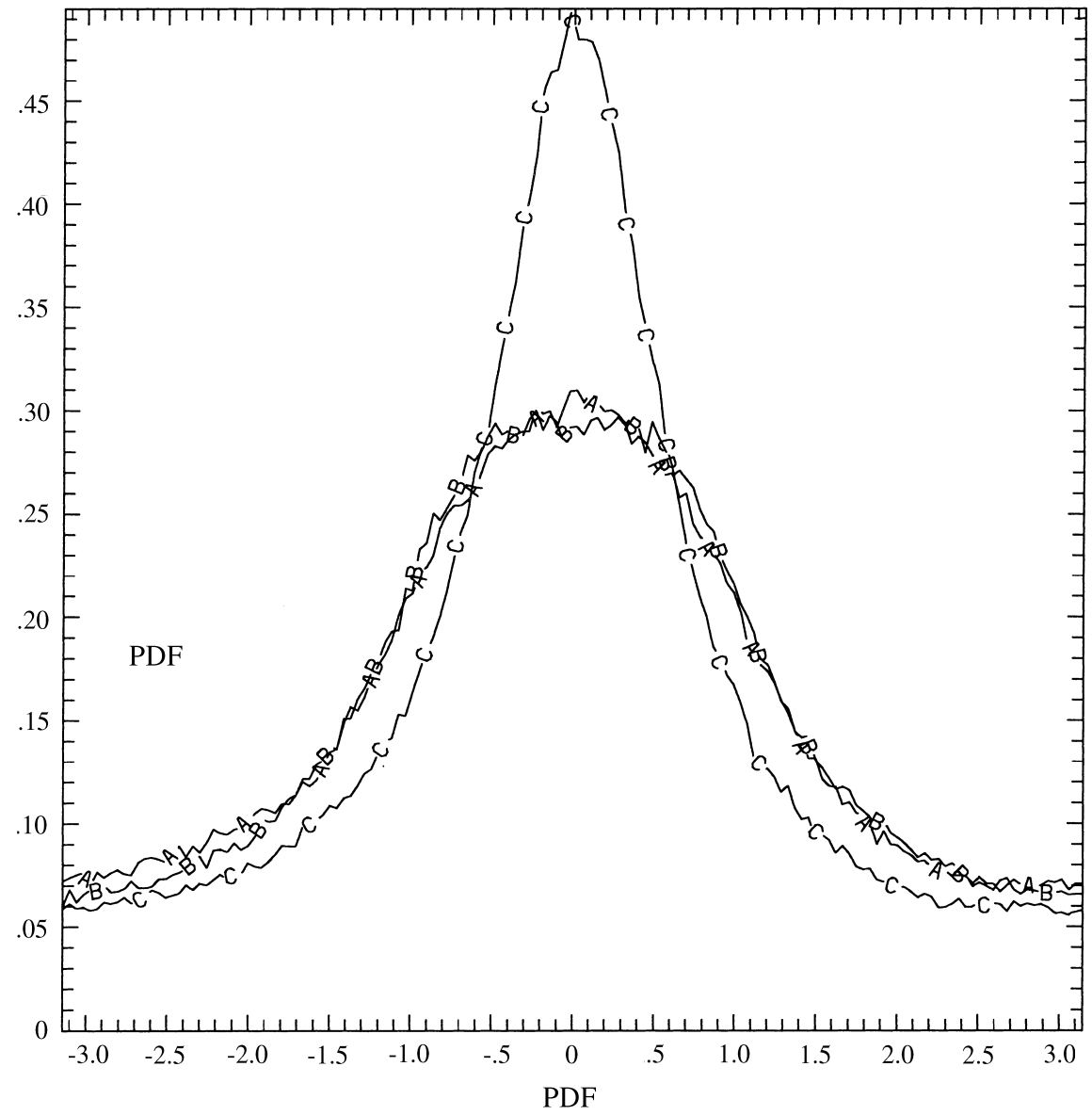

Fig. 2. PDF, conditioned by $|r| \leq 1$ and $|\nabla q|>100$, of the alignment: (A) between the tracer gradient and the compressional eigenvector $S_{-}$(i.e. $\zeta+\pi / 2$ ); (B) between the tracer gradient and the eigenvector $\boldsymbol{N}_{-}$(i.e. $\zeta-\alpha$ ); and (C) between the tracer gradient and the direction corresponding to the stable solution (3.2) (i.e. $\zeta-\zeta_{-}$). 


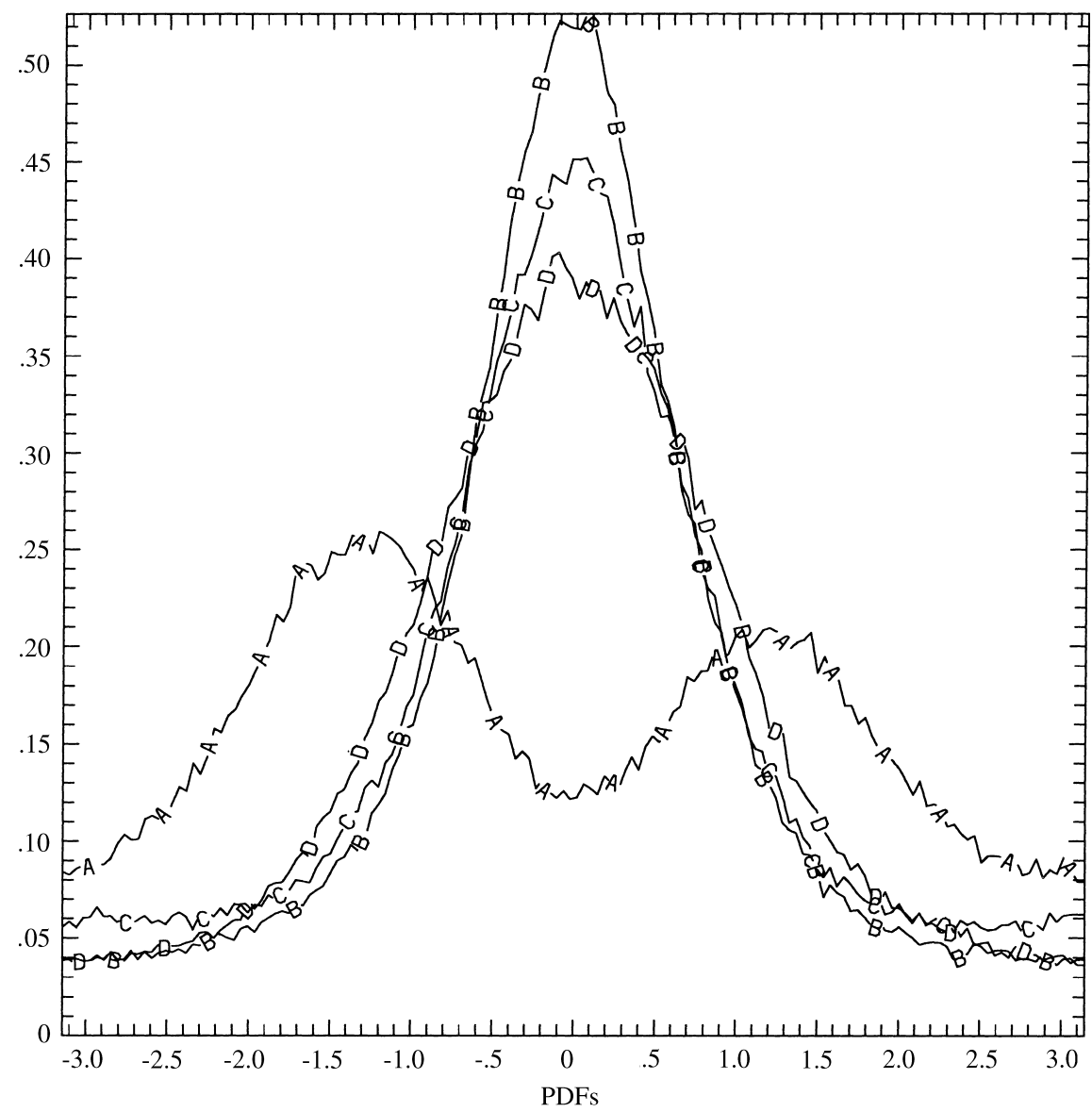

Fig. 3. PDF, conditioned by $|r|>1$ and $|\nabla q|>100$ of the alignment: (A) between the tracer gradient and the compressional eigenvector $S_{-}$ (i.e. $\zeta+\pi / 2$ ); (B) between the tracer gradient and the eigenvector $\boldsymbol{N}_{-}$(i.e. $\zeta-\alpha$ ); (C) between the tracer gradient and the eigenvector $\boldsymbol{P}_{-}^{\prime \prime}$ of the pressure Hessian (i.e. $\zeta-\delta$ ); and (D) between the tracer gradient and the preferential direction of Lapeyre et al. [13] (i.e. $\zeta-\zeta$ prob).

solution when $|r|>1$ (by checking whether $\zeta \approx$ $\left.\zeta_{\text {prob}}\right)$. At last, in order to compare our results with those of Nomura and Post [16], we have computed the statistics of the alignment of $\nabla q$ with one of the eigenvector of the pressure Hessian $\left(\boldsymbol{P}_{-}^{\prime \prime}\right)$ by considering the statistics of $\zeta-\delta$ (with $\frac{1}{2}(\delta+\pi / 2)$ the angle between $\boldsymbol{P}_{-}^{\prime \prime}$ and $\boldsymbol{S}_{-}$, see Fig. 1).

The statistical results clearly confirm the partition of the physical space into strain-dominated regions $(|r|<1)$, which concerns $60 \%$ of physical space, and "effective" rotation-dominated regions $(|r|>1)$. The key results are summarized in Figs. 2 and 3 that display the probability density function (PDF) of the different angles conditioned by $|\nabla q|>100$ (which concerns more than $50 \%$ of physical space). ${ }^{2}$

For the strain-dominated regions, the PDFs (Fig. 2) reveal that $\nabla q$ preferentially aligns with $\zeta_{-}$. The alignment with $\boldsymbol{S}_{-}$and $\boldsymbol{N}_{-}$are comparable and less efficient than with $\zeta_{-}$, but more efficient than with $\boldsymbol{P}_{-}^{\prime \prime}$ (not shown). These results clearly confirm that for the strain-dominated regions the solution proposed by Lapeyre et al. [13], i.e. $\zeta_{-}$, is the best one. On the other hand, the consideration of $\boldsymbol{N}_{-}$does not introduce more information on the gradient orientation than that of $\boldsymbol{S}_{-}$.

\footnotetext{
${ }^{2}$ The mean $|\nabla q|$-value is 162 .
} 

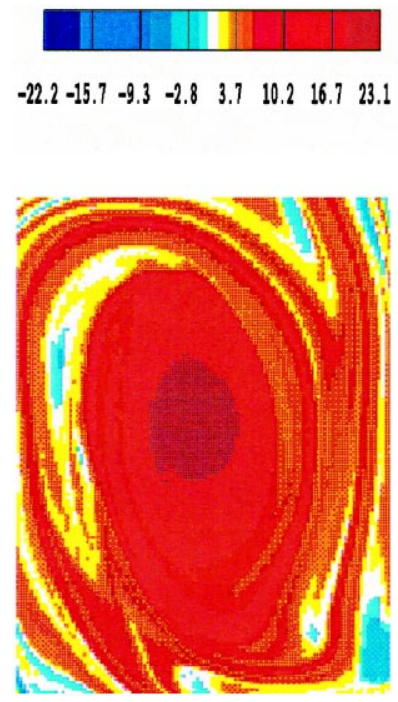

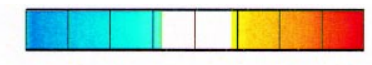

$\begin{array}{lllllllllllllllllll}-4 & -3 & -2 & -1 & 0 & 1 & 2 & 3 & 4\end{array}$

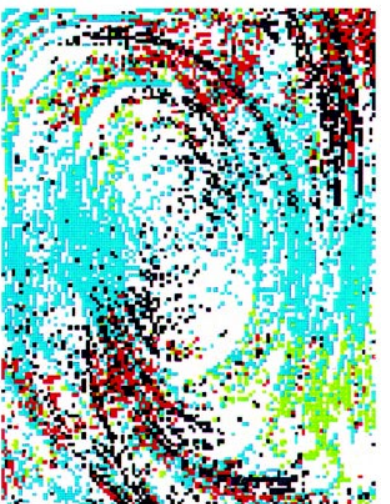

Fig. 4. Vorticity (left); $r$ (middle); alignment (with a precision of $\pm 13^{\circ}$ ) of tracer gradient vectors with $\boldsymbol{S}_{-}$(black), $\boldsymbol{N}_{-}$(blue), $\zeta_{-}$when $|r|<1$ (red) and $\zeta_{+}$when $|r|<1$ (green) (right).

For the "effective" rotation-dominated regions (Fig. 3) the results significantly differ. The alignment of $\nabla q$ with $N_{-}$strongly dominates. It is more efficient than with $\boldsymbol{P}_{-}^{\prime \prime}$ and significantly much better than with $\zeta_{\text {prob }}$. This figure shows that the tracer gradient vectors actually lie close to the bisector of the axes of the strain rate tensor with, however, a non-zero shift as displayed by the enlarged PDF of $\zeta+\zeta_{\text {prob }}$. This non-zero shift appears to be well captured by $\boldsymbol{N}_{-}$, which is a significant improvement relatively to the previous studies.

Since the alignment of $\nabla q$ in the strain-dominated regions significantly differs from that in the "effective" rotation-dominated regions, we have assessed the sharpness of the boundary $|r|=1$. For that purpose we have calculated the same PDFs as in Figs. 2 and 3 for the regions corresponding to $0.8<|r| \leq 1$ and $1<$ $|r| \leq 1.2$. The PDFs (not shown) clearly confirm the strong preference of $\nabla q$ to align with $\zeta_{-}$when $|r|<1$ and with $\boldsymbol{N}_{-}$when $|r|>1$, which emphasizes the role of $|r|=1$ as a threshold in the alignment dynamics.

Fig. 4, that focuses on one vortex of the turbulent field (Fig. 4(left)), provides an illustration of a region where both regimes, $|r|<1$ and $|r|>1$, coexist. Fig. 4(middle) shows that these regimes are well separated and that the $|r|>1$ regime is dominating.
The vortex core is a region where $r>1$ because of large values of $\omega$. The vortex periphery is composed of regions where $|r|<1$ because of large values of $\sigma$ and regions where $r<-1$ because of large values of $\mathrm{d} \phi / \mathrm{d} t$ (whose sign is opposite to that of $\omega$, Fig. 4(left)). Moreover, the $|r|>1$ region at the vortex periphery is characterized by a sharp interleaving of negative and positive values with a domination of the latter. Actually, for all vortices of the turbulent flow field, we have observed that large values of $r$ at the vortex periphery and in the vortex core globally have opposite sign. Fig. 4(right) shows the directions the tracer gradient vectors prefer to align with among the directions: $\boldsymbol{S}_{-}, \zeta_{-}, \zeta_{+}$and $\boldsymbol{N}_{-}$. Clearly there is a strong preference for $\boldsymbol{\nabla} q$ to align with $\boldsymbol{N}_{-}$, where $|r|>1$ and with $\zeta_{-}$, where $|r| \leq 1$. However, it is interesting to note that in some regions where $|r| \leq 1$ (as in the lower right edge) some gradients prefer to align with $\zeta_{+}$.

Thus the new outcome of this study concerns the "effective" rotation-dominated regions for which the present results clearly display a preferential alignment with the eigenvector $\boldsymbol{N}_{-}$, which differs from the bisector of the eigenvectors of $\boldsymbol{S}$ when $s$ is non-zero, yielding a non-zero exponential growth. This outcome has to be contrasted with previous results that only predicted a perfect alignment with the bisector of the 


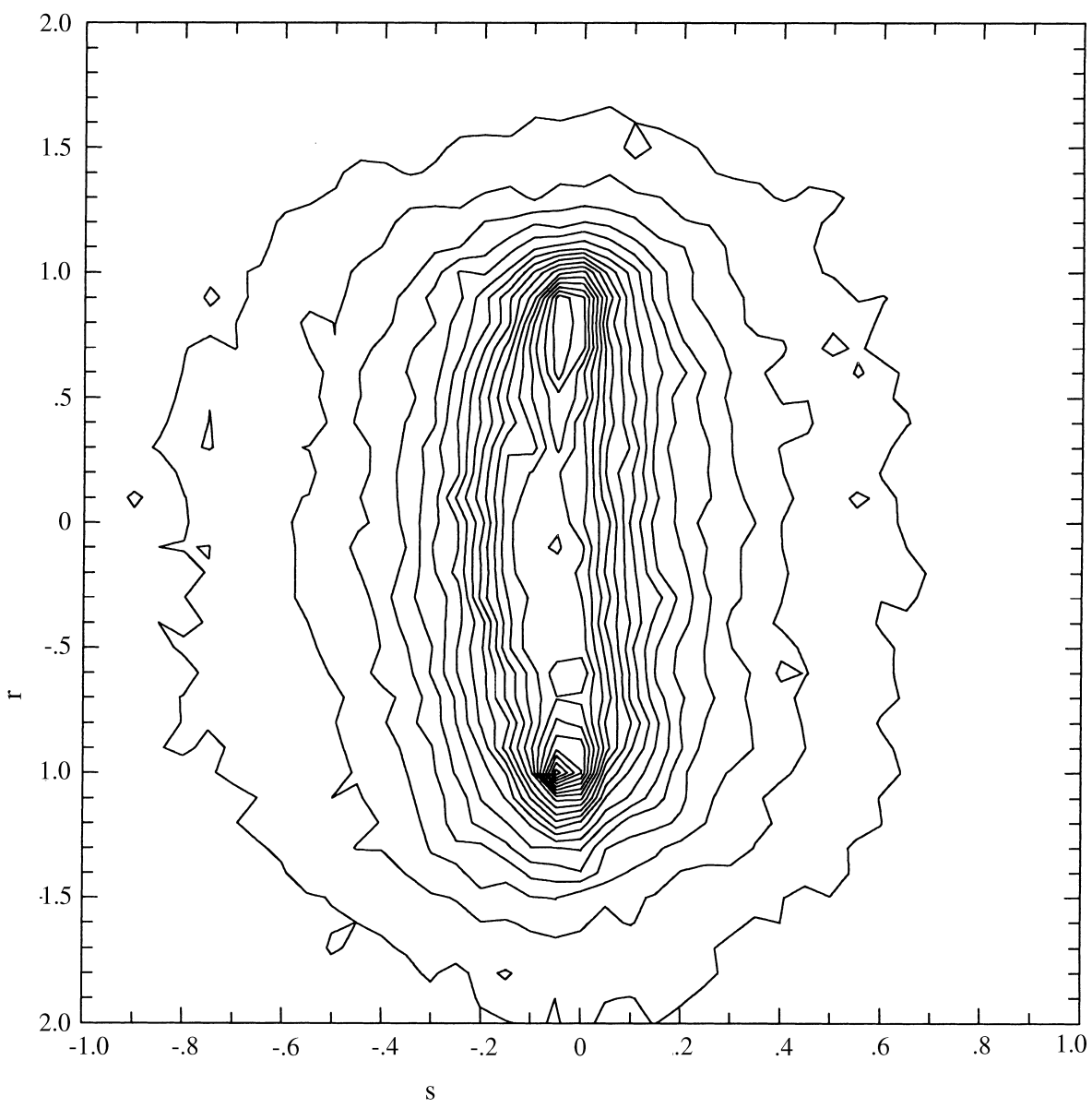

Fig. 5. Joint PDF of $s$ with $r$ conditioned by $|\nabla q|>100$.

eigenvectors of $\boldsymbol{S}$ and consequently a zero exponential growth. Therefore, the present results strongly emphasize the importance of the parameter $s$, which was not found in previous studies. Furthermore, the alignment with $\boldsymbol{N}_{-}$is better than with $\boldsymbol{P}_{-}^{\prime \prime}$ for $98 \%$ of the tracer gradients, ${ }^{3}$ which justifies the present approach using the time evolution of the tracer gradient norm rather than of the vector itself. At last this clearly confirms the significant influence of the full Lagrangian second order dynamics. On the other hand, for the strain-dominated regions, the results well confirm the conclusions of Lapeyre et al. [13] about the preferential alignment of the tracer gradient vector with $\zeta_{-}$.

\footnotetext{
${ }^{3}$ Indeed for the remaining $2 \%$ that correspond to the largest gradients, another alignment dynamics seems to prevail.
}

4.2. The role of the parameters $r$ and $s$ on the alignment process

The alignment of $\nabla q$ with either the direction of the stable solution (3.2) or with $\boldsymbol{N}_{-}$depends on the parameters $r$ and $s$. So one question is: where are the tracer gradients and the different alignment properties located in the parameter space $\{r, s\}$ ? The region where most of the tracer gradients are located is displayed by the joint PDF of $r$ and $s$ conditioned by $|\nabla q|>100$ (Fig. 5). Tracer gradients are mostly found where $|r|<1.5$ (with an almost even distribution) and where values of $s$ are slightly negative. Furthermore, we have found (figure not shown) that, in the region where $|r| \leq 1$ tracer gradients that align with the stable solution (3.2) are 


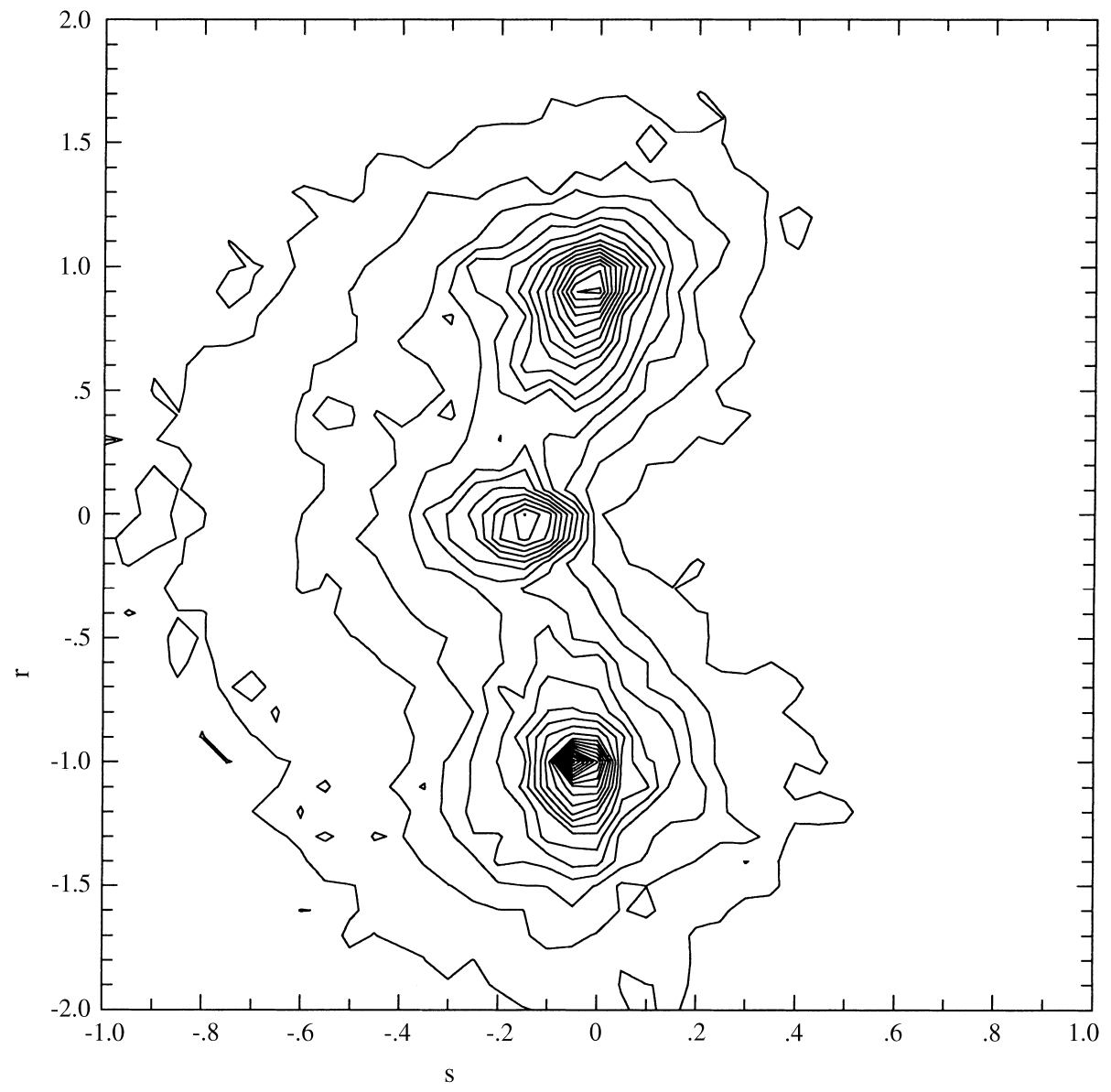

Fig. 6. Joint PDF of $s$ with $r$ conditioned by $|\nabla q|>100$ and $\cos (\zeta-\alpha)>0.9$.

also almost evenly distributed between $r=-1$ and $r=1$.

Fig. 6 shows the joint PDF of $s$ and $r$ conditioned by $|\nabla q|>100$ and $\cos (\zeta-\alpha)>0.9$ (which concerns more than $18 \%$ of physical space). This corresponds to an angle of $\nabla q$ with $N_{-}$that is less than $13^{\circ}$. It reveals that tracer gradients that align with $\boldsymbol{N}_{-}$are more frequently found in three well-defined regions whose characteristics are close to those of the first two limiting cases discussed in Section 3. The first region is centered around the point $\{r, s\}=\{0,-0.15\}$. It means that $\tan \alpha=s / r \approx \infty$ and therefore that the eigenvectors of $\boldsymbol{N}$ and $\boldsymbol{S}$ are almost parallel. This situation is close to the first limiting case (the saddle point). We have checked that, in this region, the alignment of the tracer gradient vector with the stable solution (3.2) is, however, better than with $N_{-}$. The two other regions are centered around the peaks $\{r, s\}=\{-1,-0.05\}$ and $\{r, s\}=\{0.95,-0.025\}$. This means that $\tan \alpha$ is small but non-zero in those regions and thus the angle between the eigenvectors of $\boldsymbol{N}$ and $S$ is not equal to $\pi / 4$. The consequence is that the direction of $\nabla q$ should differ from the bisector of the eigenvectors of $S$. Therefore, the tracer gradient exponential growth rate should be small but non-zero. These situations are close to the second limiting case (the axisymmetric vortices). However, they emphasize the important role of $s$. Again, we have checked that the alignment of the tracer gradient vector with the stable solution (3.2) is better than with $\boldsymbol{N}_{-}$when $|r| \leq 1$. However, when $|r|>1$, the angle of $\nabla q$ with $N_{-}$is much closer to 0 than when $|r| \leq 1$. 
The preceding results reveal that the alignment of the tracer gradient vectors with $\boldsymbol{N}_{-}$occurs preferentially for negative values of $s$. This feature is corroborated by the joint PDF (not shown) of $s$ and the growth rate of the tracer gradient norm, defined as

$\Delta=-\sigma \sin \zeta$

which displays a negative correlation of $s$ with $\Delta$ and which presents a strong asymmetry that is more pronounced for large amplitudes of $s$ : positive (negative) values of the growth rate are more frequently found in regions where $s$ is negative (positive). Thus, when the amplitude of $s$ is large, the sign of $s$ affects the alignment process and the growth rate of the tracer gradient norm. On the other hand, the sign of $r$ does not seem to have a so much pronounced influence on the alignment of $\nabla q$ with the eigenvectors of $N$ (and therefore on the sign of the growth rate). We observe, however, in Fig. 6 that the PDF is stronger in the region centered around $\{r, s\}=\{-1,-0.05\}$ than in the region around $\{r, s\}=\{0.95,-0.025\}$.

\subsection{Consequence on the growth rate of the tracer gradient}

What can be inferred from the alignment properties, in particular in the "effective" rotation-dominated regions, for the production of the tracer gradients and the tracer cascade? One way to address this question is to compare the observed instantaneous growth rates of the tracer gradient norm $(\Delta)$ with their estimations using the strain rate amplitude and the

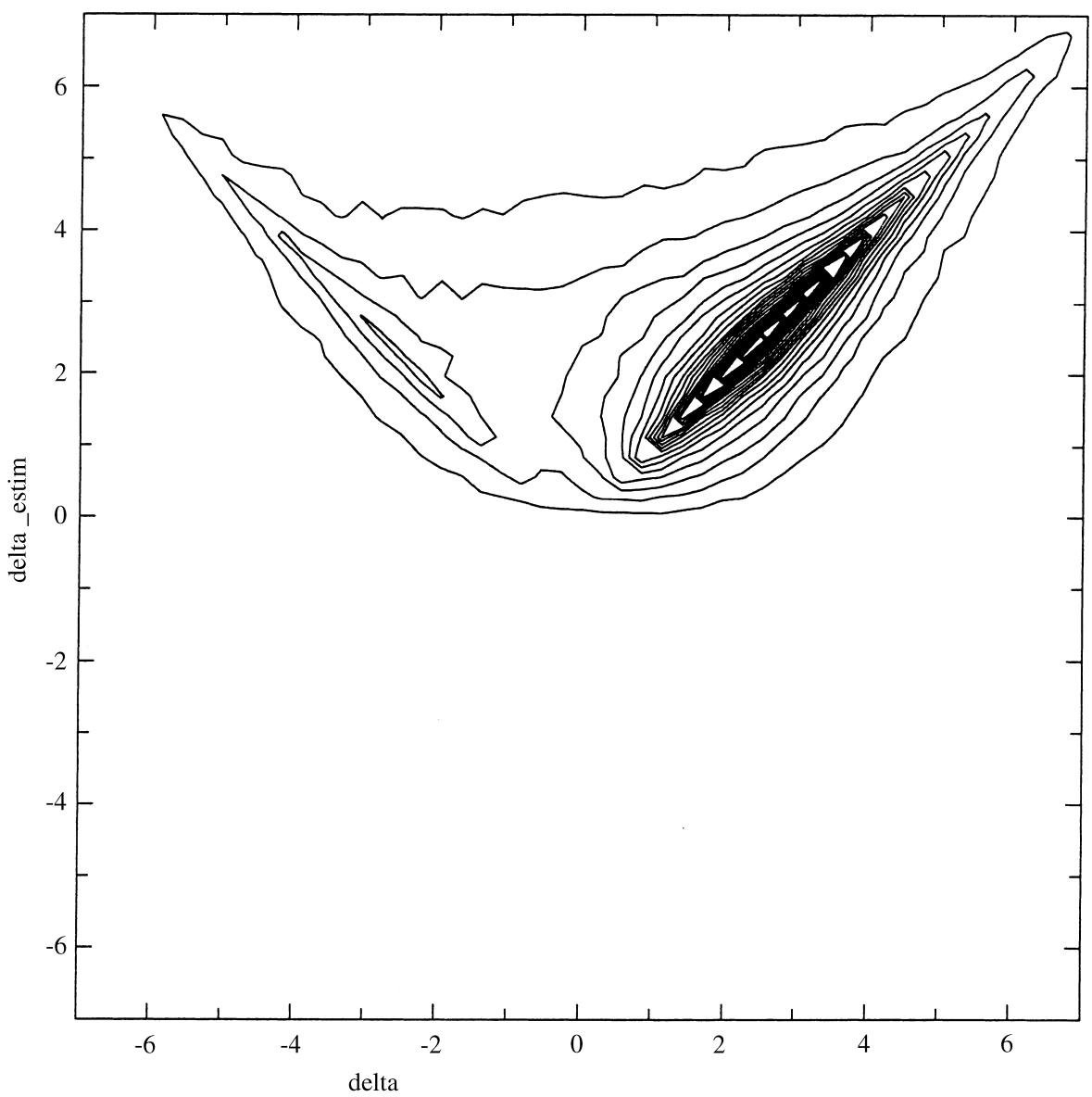

Fig. 7. Joint PDF of the growth rate with its estimation using (4.2) and conditioned by $|\nabla q|>100$ and $|r|<1$. 


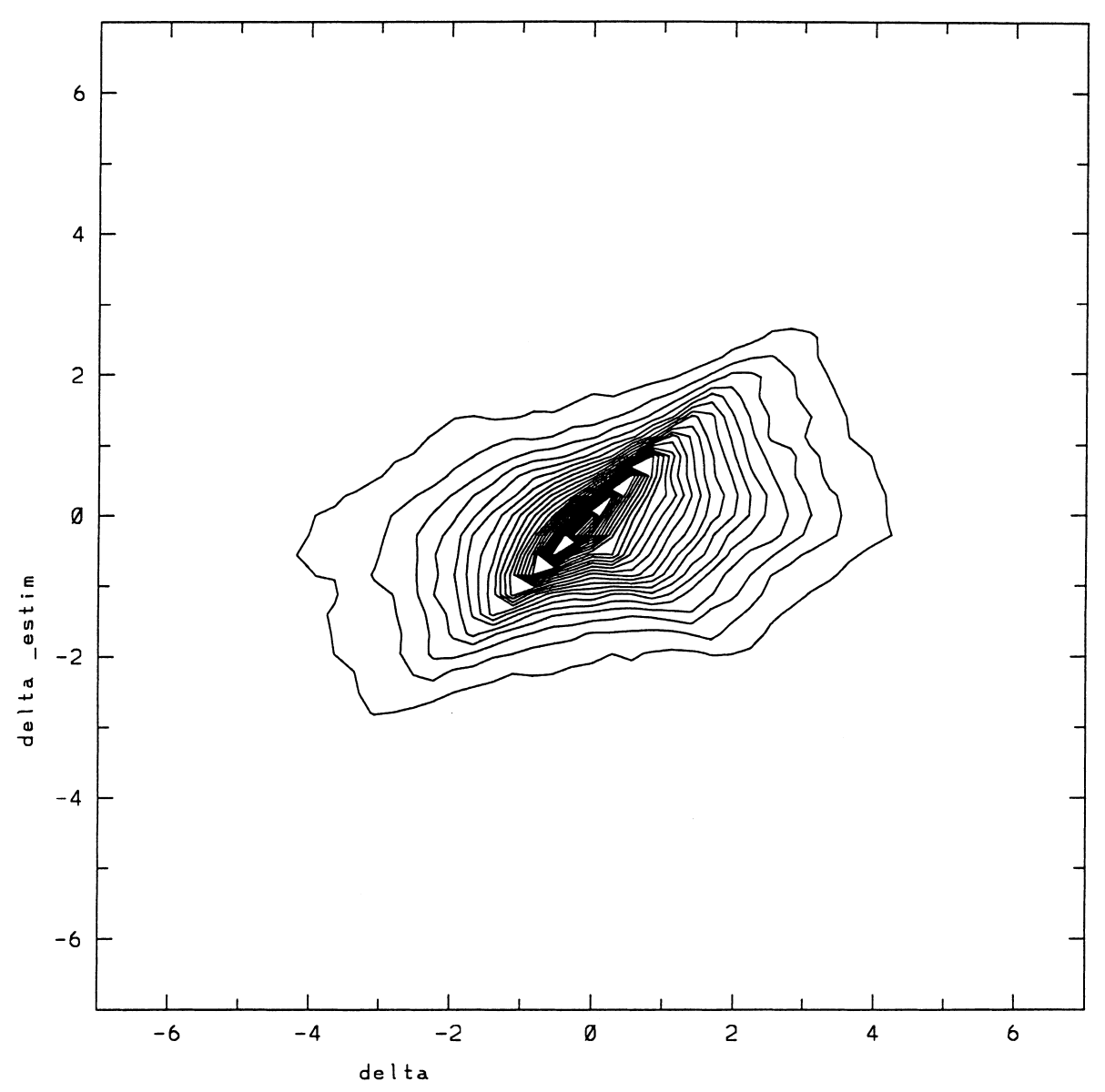

Fig. 8. Same as Fig. 7 but using (4.3) and conditioned by $|r|>1$.

analytical expressions for the alignment properties. The strong preference for $\nabla q$ to be aligned with $\zeta_{-}$ in the strain-dominated regions and with $N_{-}$in the "effective" rotation-dominated regions leads to the following analytical expressions of the instantaneous exponential growth rate, $\Delta$, of the tracer gradient magnitude:

$\Delta=\sigma \sin (\arccos r)=\sigma \sqrt{1-r^{2}}$ when $|r| \leq 1$,

$\Delta=-\sigma \sin (\alpha)=-\sigma \frac{s}{\chi} \quad$ when $|r|>1$.

The joint PDFs of the experimental values of $\Delta$ with the analytical expressions provided by (4.2) and (4.3), shown in Figs. 7 and 8, reveal that these estimations of the growth rate are statistically close to the experi- mental values. In the regions where $|r| \leq 1$, the joint PDF (Fig. 7) exhibits a maximum near $\Delta=2.5$. Most of the tracer gradients located in those regions align with $\zeta_{-}$. However, there is a branch in the upper left-hand quarter that corresponds to a correlation of -1 . This means that, at those locations, the tracer gradient vectors align with $\zeta_{+}$instead of $\zeta_{-}$. So far we have no explanation for this intriguing feature. Fig. 8 reveals that in the "effective" rotation-dominated regions, the growth rate of the tracer gradients ranges between -1.5 and 1.5 and that the estimation produced by (4.3) is statistically correct. These results again emphasize the important role of the amplitude and sign of $r$ and $s$ on the growth rate of the tracer gradients and therefore on the stirring properties of a $2 \mathrm{D}$ flow field. Thus the main novel feature that emerges, relatively 
to the previous studies, is that taking into account the rate of change of the strain rate amplitude allows to predict the exponential growth rate of the tracer gradients in the "effective" rotation-dominated regions.

\section{Conclusion}

The purpose of this study was to examine the alignment dynamics of the tracer gradient vectors by considering the second order Lagrangian dynamics. In accordance with the results of Hua and Klein [9], the approach used has been to consider the first two order equations for the tracer gradients. The equations for the tracer gradient norm are considered instead of those for the tracer gradient vectors. These equations are indeed independent of the coordinates system and allow to focus on the geometrical alignment of the tracer gradient vectors relatively to the strain eigenvectors. The main result, that emerges from direct numerical simulations, is that the full consideration of the second order Lagrangian dynamics significantly improves the statistical estimation of the alignment dynamics and therefore the knowledge of the stirring properties of a 2D turbulent flow field.

More precisely, defining a parameter $r$, that measures the competition of the "effective" rotation effects (due to both the vorticity and the rotation of the strain axes) with the strain modulus effects, the present results well confirm those obtained in [13] in the strain-dominated regime $(|r|<1)$ : for this regime, that concerns almost $60 \%$ of the physical space, there is a stronger preference for the alignment with a direction corresponding to a steady equilibrium between the "effective" rotation and strain effects.

The major improvement which emerges from our approach concerns the "effective" rotation-dominated regime $(|r|>1)$ that concerns $40 \%$ of the physical space: the experimental results clearly reveal a strong preference for the tracer gradient vectors to be statistically aligned with $N_{-}$, one of the eigenvectors of a matrix $N$ related to the pressure Hessian. These results are corroborated by other numerical simulations of decaying 2D turbulence (not reported here). The eigenvector $N_{-}$differs from the bisector of the eigenvectors of $S$ when the time evo- lution of the strain rate modulus, $s$, is non-zero. This feature strongly emphasizes the importance of this parameter $s$, which was not found in the previous studies. The consequence is that, in these "effective" rotation-dominated regions, the non-zero exponential growth or decay of the tracer gradient vector (and therefore both the sign and amplitude of the growth rate) can be predicted contrary to previous results which predicted zero growth and only a rotation of this vector.

The tensor $\boldsymbol{N}$ is directly related to the time evolution of the amplitude and orientation of the strain tensor. This clearly confirms the significant influence of the pressure Hessian, more precisely of its anisotropic part, since this part modifies both the amplitude and orientation of the strain tensor. Furthermore, this emphasizes the strong influence of the non-local (in terms of the velocity field) interactions on the alignment dynamics in particular for the "effective" rotation-dominated regime. Indeed calculation of the anisotropic part of the pressure Hessian requires to solve a Poisson equation over the whole domain [9], whereas the isotropic part is expressed in terms of the local velocity gradients.

The present results are similar to those obtained recently by Nomura and Post [16] for 3D turbulence. For the rotation-dominated regions, these authors found that the vorticity vector (equivalent to the tracer gradient vector in 2D turbulence) preferentially aligns with one of the pressure Hessian eigenvectors. Our results clearly reveal that for a $2 \mathrm{D}$ field, the tracer gradients prefer to align with $\boldsymbol{N}_{-}$rather than with the eigenvectors of the pressure Hessian, which justifies the present approach using the equations for the tracer gradient norm. An explanation of this difference is that in a 2D flow field, the tracer gradients are affected by the vorticity (see Eq. (A.1)), whereas in a $3 \mathrm{D}$ field the vorticity vector cannot be directly affected by itself. This is why the expression of $\boldsymbol{N}$ (see (A.6)) involves, besides the pressure Hessian $\boldsymbol{P}^{\prime \prime}$ and its isotropic part $\left(\lambda_{0} I\right)$, an additional term linearly related to the vorticity $\left(\boldsymbol{\Omega} \nabla \boldsymbol{u}^{*}\right)$.

It should be mentioned that throughout this study we do not make distinction between the gradient of a passive tracer conserved on a Lagrangian trajectory 
and that of the vorticity. We have indeed found in our numerical 2D simulations that, for times longer than an initial enstrophy time scale, the two gradients have an identical orientation in most of the domain. This is also confirmed by Babiano et al. [1] who have noted that isolines of tracer and vorticity have similar orientations. Therefore, the present results are valid for both gradients. However, a more thorough study on the comparison between the behaviors of active and passive scalars in 2D turbulence is under progress.

Two questions arise from the results of the present study. The first one concerns the sign of the growth rate of the tracer gradient. Indeed the analytical findings of this study indicate that in the strain-dominated regions, the tracer gradient vectors should align in a direction that corresponds to a positive growth rate. However, at some locations, the numerical results clearly reveal an alignment just in the opposite direction. This suggests that at those locations the tracer gradient vectors preferentially align in the direction of the unstable fixed point of (3.1). The mechanisms that force this solution to be stabilized in those regions, and more specifically the role of the parameter $s$, should be investigated as $s$ was not taken into account in the derivation of (3.2) and (3.3). The second question concerns the effects of the dissipation on the alignment dynamics which have not been examined in this study. Some recent results show that both the magnitude and the form of the viscosity (using a Newtonian or an hyperviscous model) can have a significant effect on the alignment dynamics with the strain eigenvectors [22] and on the stripping of vortex caused by the steep vorticity gradients [14]. These results suggest to study more carefully the effects of the viscosity on the topology of the stirring processes. Such a study could use a recent technique of Lagrangian path analysis to take into account the diffusion on Lagrangian trajectories as it permits to have an exact expression for the tracer distribution (see for instance [5] and references therein). We intend to address these questions in the next future.

\section{Acknowledgements}

We are grateful to Xavier Carton and Raymond Pierrehumbert for their insightful suggestions and discussions on the paper. This work is supported by IFREMER and the CNRS. The calculations reported here were done on the CRAY C90 of the IDRIS (Orsay, France).

\section{Appendix A}

One approach to study the deformation of the tracer field is to consider the first two order time derivative equations of its gradient vectors (see $[3,9]$ ):

$$
\begin{aligned}
\frac{\mathrm{d} \boldsymbol{\nabla} q}{\mathrm{~d} t} & =-\boldsymbol{\nabla} \boldsymbol{u}^{*} \boldsymbol{\nabla} q, \\
\frac{\mathrm{d}^{2} \boldsymbol{\nabla} q}{\mathrm{~d} t^{2}} & =\left[\left[\boldsymbol{\nabla} \boldsymbol{u}^{*}\right]^{2}-\frac{\mathrm{d} \boldsymbol{\nabla} \boldsymbol{u}^{*}}{\mathrm{~d} t}\right] \nabla q,
\end{aligned}
$$

with $\boldsymbol{\nabla}$ the gradient operator and $\boldsymbol{\nabla} \boldsymbol{u}^{*}$ the transpose of the velocity gradient tensor, expressed as

$$
\begin{aligned}
\boldsymbol{\nabla} \boldsymbol{u}^{*} & =\left[\begin{array}{ll}
\partial_{x} u & \partial_{x} v \\
\partial_{y} u & \partial_{y} v
\end{array}\right] \\
& =\frac{1}{2}\left[\begin{array}{cc}
\sigma_{\mathrm{n}} & \sigma_{\mathrm{s}} \\
\sigma_{\mathrm{s}} & -\sigma_{\mathrm{n}}
\end{array}\right]+\frac{1}{2}\left[\begin{array}{cc}
0 & \omega \\
-\omega & 0
\end{array}\right]=\boldsymbol{S}+\boldsymbol{\Omega},
\end{aligned}
$$

where $\omega, \sigma_{\mathrm{n}}$, and $\sigma_{\mathrm{s}}$ are, respectively, the vorticity and the normal and shear strain rates and $\boldsymbol{\Omega}=\frac{1}{2} \omega \boldsymbol{R}(\pi / 2)$ with $\boldsymbol{R}(\pi / 2)$ the rotation matrix of $\pi / 2$. Furthermore, if $\boldsymbol{P}^{\prime \prime}$ designates the Hessian matrix of the pressure:

$$
P^{\prime \prime} \equiv\left[\begin{array}{ll}
\partial_{x x} p & \partial_{x y} p \\
\partial_{x y} p & \partial_{y y} p
\end{array}\right]
$$

we have the relation [9]:

$$
\left[\left[\nabla \boldsymbol{u}^{*}\right]^{2}-\frac{\mathrm{d} \boldsymbol{\nabla} \boldsymbol{u}^{*}}{\mathrm{~d} t}\right]=-\boldsymbol{R}\left(-\frac{\pi}{2}\right) \boldsymbol{P}^{\prime \prime} \boldsymbol{R}\left(\frac{\pi}{2}\right) .
$$

This relation emphasizes the role of the pressure Hessian (i.e. of the acceleration gradient tensor) on the evolution of the tracer gradient vectors. For a 2D flow, we have

$$
\left[\left[\nabla \boldsymbol{u}^{*}\right]^{2}-\frac{\mathrm{d} \boldsymbol{\nabla} \boldsymbol{u}^{*}}{\mathrm{~d} t}\right]=\lambda_{0} \boldsymbol{I}-\frac{\mathrm{d} \boldsymbol{S}}{\mathrm{d} t},
$$

since $\mathrm{d} \omega / \mathrm{d} t=0$ and $\left[\nabla \boldsymbol{u}^{*}\right]^{2}=\lambda_{0} \boldsymbol{I}$ with $\boldsymbol{I}$ the identity matrix and $\lambda_{0}=\frac{1}{4}\left(\sigma_{\mathrm{n}}^{2}+\sigma_{\mathrm{s}}^{2}-\omega^{2}\right)$. The part $\lambda_{0} \boldsymbol{I}$ is related to the isotropic part of the pressure Hes$\operatorname{sian}\left(\lambda_{0}=-\frac{1}{2} \nabla^{2} p\right)$, whereas $\mathrm{d} \boldsymbol{S} / \mathrm{d} t$ is related to the 
non-isotropic part, which points out its non-local nature [9]. The studies of Basdevant and Philipovitch [3] and Hua and Klein [9] have clearly revealed the necessity to take into account both $\lambda_{0} \boldsymbol{I}$ and $\mathrm{d} \boldsymbol{S} / \mathrm{d} t$ for a better characterization of the stirring processes.

The tensor $\boldsymbol{N}$ defined in (2.5) can be directly related to the pressure Hessian using the preceding expressions:

$N=2\left[-R\left(-\frac{\pi}{2}\right) P^{\prime \prime} R\left(\frac{\pi}{2}\right)+\nabla u \nabla u^{*}\right]$,

or

$\boldsymbol{N}=2\left[-\boldsymbol{R}\left(-\frac{\pi}{2}\right) \boldsymbol{P}^{\prime \prime} \boldsymbol{R}\left(\frac{\pi}{2}\right)+\lambda_{0} \boldsymbol{I}-2 \boldsymbol{\Omega} \nabla \boldsymbol{u}^{*}\right]$.

\section{Appendix B}

Simpler expressions for both $S$ and $\boldsymbol{N}$ can be obtained when they are rewritten in the strain coordinates. Using the relations $\left(\sigma_{\mathrm{s}}, \sigma_{\mathrm{n}}\right)=\sigma(\cos 2 \phi, \sin 2 \phi)$ with $\sigma \geq 0$, these expressions are

$$
\begin{aligned}
& \boldsymbol{S}=\frac{\sigma}{2} \boldsymbol{R}\left(\phi-\frac{\pi}{4}\right)\left[\begin{array}{cc}
1 & 0 \\
0 & -1
\end{array}\right] \boldsymbol{R}\left(\frac{\pi}{4}-\phi\right), \\
& \boldsymbol{N}=\sigma^{2} \boldsymbol{R}\left(\phi-\frac{\pi}{4}\right)\left[\begin{array}{cc}
1-s & r \\
r & 1+s
\end{array}\right] \boldsymbol{R}\left(\frac{\pi}{4}-\phi\right),
\end{aligned}
$$

where the terms $r$ and $s$ are defined by (2.9).

\section{References}

[1] A. Babiano, C. Basdevant, B. Legras, R. Sadourny, Vorticity and passive-scalar dynamics in two-dimensional turbulence, J. Fluid Mech. 183 (1987) 379-397.

[2] M.G. Balluch, P.H. Haynes, Quantification of lower stratospheric mixing processes using aircraft data, J. Geophys. Res. 102 (D19) (1997) 23487-23504.

[3] C. Basdevant, T. Philipovitch, On the validity of the "Weiss criterion" in two-dimensional turbulence, Physica D 73 (1994) $17-30$.

[4] G.K. Batchelor, Small-scale variation of convected quantities like temperature in turbulent fluid. Part 1. General discussion and the case of small conductivity, J. Fluid Mech. 5 (1959) 113-133.

[5] M. Chertkov, G. Falkovich, I. Kolokolov, M. Vergassola, Small-scale turbulent dynamo, Phys. Rev. Lett. 83 (1999) 4065-4068.
[6] E. Dresselhaus, M. Tabor, The stretching and alignment of material elements in general flow fields, J. Fluid Mech. 236 (1991) 415-444.

[7] D.G. Dritschel, P.H. Haynes, M.N. Juckes, T.G. Shepherd, The stability of a two-dimensional vorticity filament under uniform strain, J. Fluid Mech. 230 (1991) 647-665.

[8] B. Galanti, J.D. Gibbon, M. Heritage, Vorticity alignment results for the three-dimensional Euler and Navier-Stokes equations, Nonlinearity 10 (1997) 1675-1694.

[9] B.L. Hua, P. Klein, An exact criterion for the stirring properties of nearly two-dimensional turbulence, Physica D 113 (1998) 98-110.

[10] B.L. Hua, P. Klein, G. Lapeyre, Formation of tracer gradients in nearly two-dimensional flows, Proceedings Conference on Developments in Geophysical Turbulence, Boulder, June 1998 , in press.

[11] B.L. Hua, J.C. McWilliams, P. Klein, Lagrangian acceleration in geostrophic turbulence, J. Fluid Mech. 35 (1998) 122.

[12] N.K.-R. Kevlahan, M. Farge, Vorticity filaments in two-dimensional turbulence: creation, stability and effect, J. Fluid Mech. 346 (1997) 49-76.

[13] G. Lapeyre, P. Klein, B.L. Hua, Does the tracer gradient vector align with the strain eigenvectors in $2 \mathrm{D}$ turbulence? Phys. Fluids 11 (1999) 3729-3737.

[14] A. Mariotti, B. Legras, D.G. Dritschel, Vortex stripping and the erosion of coherent structures in two-dimensional flows, Phys. Fluids 6 (12) (1994) 3954-3962.

[15] J.C. McWilliams, The emergence of isolated coherent vortices in turbulent flows, J. Fluid Mech. 198 (1984) 199-230.

[16] K.K. Nomura, G.K. Post, The structure and dynamics of vorticity and rate of strain in incompressible homogeneous turbulence, J. Fluid Mech. 377 (1998) 65-97.

[17] K. Ohkitani, Some mathematical aspects of 2D vortex dynamics, in: D. Chae (Ed.), Proceedings of Partial Differential Equations and Applications, June 1995, Seoul National University, Lecture Notes, No. 38.

[18] K. Ohkitani, S. Kishiba, Nonlocal nature of vortex stretching in an inviscid fluid, Phys. Fluids 7 (2) (1995) 411-421.

[19] A. Okubo, Horizontal dispersion of floatable particles in the vicinity of velocity singularities such as convergences, Deep-Sea Res. 17 (1970) 445-454.

[20] R.T. Pierrehumbert, H. Yang, Global chaotic mixing on isentropic surfaces, J. Atmos. Sci. 50 (1993) 2462-2480.

[21] A. Provenzale, Transport by coherent barotropic vortices, Annu. Rev. Fluid Mech. 31 (1999) 55-93.

[22] B. Protas, A. Babiano, K.R. Kevlahan, On geometrical alignment properties of two-dimensional forced turbulence, Physica D 128 (1999) 169-179.

[23] A. Tsinober, M. Ortenberg, L. Shtilman, On depression of nonlinearity in turbulence, Phys. Fluids 11 (8) (1999) 22912297.

[24] J. Weiss, The dynamics of enstrophy transfer in twodimensional turbulence, Physica D 48 (1991) 273-294.

[25] J. Weiss, Report LJI-TN-81-121, La Jolla Institute, San Diego, CA, 1981. 